\section{Difficulty in Diagnosing Surgical Site Infection After Arthroscopy in Developing Countries}

To the Editor-In the United States, the use of arthroscopy has been increasing since the 1970s.' Although infectious complications after arthroscopy appear to be fairly rare $(0.01 \%-0.48 \%$ of procedures $)$, significant morbidity and significant costs are associated with the procedure. ${ }^{2}$ In Thailand, although arthroscopy has been increasingly performed during the past decade, it has not been incorporated into the surveillance systems of most Thai hospitals, ${ }^{3}$ and postdischarge surveillance is often suboptimal. ${ }^{4}$ The lack of a national benchmark also makes it difficult to compare rates of surgical site infection (SSI) after arthroscopy between hospitals. We report on the difficulty of using Centers for Disease Control and Prevention (CDC) definitions ${ }^{5}$ to diagnose SSI after arthroscopy and highlight some implications for surveillance in developing countries.

In September 2008, an infection control unit was notified of 4 potential cases of SSI after arthroscopy at a hospital in Thailand; all cases met the definitions for postoperative infections-these definitions were introduced into hospitals participating in the CDC National Nosocomial Infections Surveillance (NNIS) system-and occurred in $2008 .{ }^{5}$ An outbreak investigation was initiated in September 2008. In this particular hospital, 6 surgeons performed this type of surgery. The surgical logbook was reviewed to identify all patients who had undergone arthroscopy, followed by a careful chart review, for evidence of postoperative SSI. A line list was created. Postdischarge surveillance (by mail and/or telephone) was also performed for all patients who had undergone arthroscopy during the period from January through November 2008, according to the CDC-NNIS recommendations. ${ }^{5}$ Infection control practices were observed in the operating room of the orthopedic ward (for suboptimal hand hygiene or lapses in sterile techniques or in the implementation of some other infection control measure).

There were 293 arthroscopic procedures performed during the period from January through November 2008. A total of 6 suspected cases of SSI after arthroscopy were identified. However, after a careful review of the medical records, only 3 of these 6 cases showed evidence of SSI. Three $(50 \%)$ of the 6 original suspected cases did not show evidence of infection. An additional case of SSI after arthroscopy was identified during postdischarge surveillance. Notably, the treating physicians had diagnosed all 4 cases of SSI. Each of the 4 patients required a 6 -week course of systemic antibiotic prophylaxis. The median age of the patients was 26 years (range,
23-31 years), and the median duration of surgery was 5 hours (range, 4-6 hours): 2 (50\%) of the 4 patients were operated on by doctor $\mathrm{A}$, and $2(50 \%)$ had material implanted in the joint space. Joint fluid specimens from each patient were obtained for culture, and these specimens revealed that 1 patient was infected with methicillin-resistant Staphylococcus aureus and that another was infected with coagulase-negative Staphylococcus species; 2 of the 4 patients' joint fluid specimens did contain a microorganism. Two (50\%) of the 4 patients were readmitted to the hospital for multiple surgical joint procedures. Compared with patients who did not develop an SSI after arthroscopy, patients who did develop an SSI after arthroscopy were more likely to have a prolonged duration of surgery (median duration, 1.4 vs 5 hours; $P=$ .04 ). There were no differences in other preoperative, operative, and postoperative risk factors between patients who developed an SSI after arthroscopy and patients who did not. The rates of SSI after arthroscopy and the infection control practices observed are summarized in the Table for each surgeon. After the investigation, feedback was provided to the surgeons, and educational sessions were held for healthcare personnel who worked in the operating rooms.

Because of the low incidence of SSI after arthroscopy, ${ }^{1,2}$ studies that try to delineate the risk factors for SSI and/or that try to describe the effects of the implementation of preventive measures during orthopedic surgery are challenging.

TABLE. Data on the Rates of Surgical Site Infection (SSI) After Arthroscopy and the Infection Control Practices Observed at a Hospital in Thailand in 2008, by Surgeon

\begin{tabular}{lcc}
\hline Surgeon & $\begin{array}{c}\text { SSI rate, cases per } \\
100 \text { procedures }\end{array}$ & Observation(s) \\
\hline Doctor A & 2.2 & $\begin{array}{c}\text { Suboptimal hand washing } \\
\text { between procedures, lapses } \\
\text { in sterile technique, and } \\
\text { high traffic during } \\
\text { procedure } \\
\text { Suboptimal hand washing } \\
\text { between procedures, lapses } \\
\text { in sterile technique, high } \\
\text { traffic during procedure }\end{array}$ \\
Doctor B & 0.51 & $\begin{array}{c}\text { Suboptimal hand washing } \\
\text { between procedures, high } \\
\text { traffic during procedure }\end{array}$ \\
Doctor C & 0.0 & High traffic during procedure \\
Doctor E & 0.0 & High traffic during procedure \\
Doctor F & 0.0 & High traffic during procedure
\end{tabular}

NOTE. Suboptimal hand washing was defined as either a lack of hand washing before or after surgery, a handwashing duration of $<3$ minutes, or a failure to follow hygienic handwashing procedures. Lapses in sterile technique were defined as the touching of nonsterile items during surgery. High traffic during procedure was determined by counting the number of times that healthcare personnel opened the door of the operating room. 
Our report suggests that the CDC-NNIS definition is not specific. The sensitivity of the CDC-NNIS definition for SSI is further compromised in developing countries, where postdischarge surveillance is performed in a suboptimal manner (ie, surveillance consists of a doctor reviewing the patient's medical records or it occurs during hospital readmission). Interestingly, the specificity of diagnosis increased when a physician's diagnosis of infection was taken into account. In addition, we identified prolonged duration of surgery as a potential risk factor for SSI after arthroscopy, as has been shown for cases of SSI after total knee arthroplasty. ${ }^{6}$ Given the burden of significant morbidity and significant costs associated with SSI after arthroscopy, ongoing surveillance, a rapid response to increased rates of infection, coordinated efforts by surgeons and healthcare personnel, and adherence to basic infection control guidelines are essential to improve patient outcomes and decrease infection rates in developing countries.

\section{ACKNOWLEDGMENTS}

Financial support. Our study was partially funded by the Thammasat University Infectious Diseases and Infection Control Research Unit (to A.A.).

Potential conflicts of interest. All authors report no conflicts of interest relevant to this article.

Anucha Apisarnthanarak, MD;

Siriporn Wittayachanyapong, MD; Pranee Sitaposa, MD; Kanokporn Thongphubeth, RN; Hilary Babcock, MD; Victoria J. Fraser, MD
From the Division of Infectious Diseases and Infection Control, Thammasat University Hospital, Pratumthani (A.A., K.T.), the Department of Medicine, Khon Khan University, Khon Khan (S.W.), and the Department of Pediatrics, Queen Sirikit National Institute of Child Health, Bangkok (P.S.), Thailand; and the Division of Infectious Diseases, Washington University School of Medicine, Saint Louis, Missouri (H.B., V.J.F.).

Address reprint requests to Anucha Apisarnthanarak, MD, Division of Infectious Diseases, Thammasat University Hospital, Pratumthani, Thailand 12120 (anapisarn@yahoo.com). Infect Control Hosp Epidemiol 2009; 30:609-610

(C) 2009 by The Society for Healthcare Epidemiology of America. All rights reserved. 0899-823X/2009/3006-0021\$15.00. DOI: 10.1086/597265

\section{REFERENCES}

1. Jackson RW. Arthroscopic surgery. J Bone Joint Surg Am 1983; 65:416420.

2. Babcock HM, Matava MJ, Fraser V. Postarthroscopy surgical site infections: review of the literature. Clin Infect Dis 2002; 34:65-71.

3. Narong MN, Thongpiyapoom S, Thaikul N, Jamulitrat S, Kasatpibal N. Surgical site infections in patients undergoing major operations in a university hospital: using standardized infection ratio as a benchmarking tool. Am J Infect Control 2003; 31:274-279.

4. Kasatpibal N, Jamulitrat S, Chongsuvivatwong V. Standardized incidence rates of surgical site infection: a multicenter study in Thailand. Am J Infect Control 2005; 33:587-594.

5. Garner JS, Jarvis WR, Emori TG, Horan TC, Hughes JM. CDC definitions for nosocomial infections, 1988. Am J Infect Control 1988; 16:128-140.

6. Peersman G, Laskin R, Davis J, Peterson MG, Richart T. Prolonged operative time correlates with increased infection rate after total knee arthroplasty. HSS J 2006; 2:70-72. 